\title{
IDENTIFICATION STRATEGY OF ANISOTROPIC BEHAVIOR LAWS: APPLICATION TO THIN SHEETS OF ALUMINIUM A5
}

\author{
Amna Znaidi, Olfa Daghfas \\ National School of Engineers of Tunis, Departement of Mechanical Engineering, Tunis, Tunisie \\ e-mail: amna.znaidi@Laposte.net; daghfasolfa@yahoo.fr
}

Amen Gahbiche

National School of Engineers of Monastir, Mechanical Engineering Laboratory, Monastir, Tunisie e-mail: amen_gahbiche@yahoo.fr

RACHID NASRI

National School of Engineers of Tunis, Departement of Mechanical Engineering, Tunis, Tunisie

e-mail: rachid.nasri@enit.rnu.tn

\begin{abstract}
Numerical simulation provides a valuable assistance in the controlling of forming processes. The elasto-plastic orthotropic constitutive law is based on the choice of an equivalent stress, a hardening law and a plastic potential. An identification of the model parameters from an experimental database is developed. This database consists in hardening curves and Lankford coefficients of specimens subjected to off-axis tensile tests. The proposed identification strategy is applied to aluminum sheets. The behavior of this material is studied under several solicitations. The anisotropic behavior of the aluminum plate is modeled using the Barlat criterionand the hardening law. The obtained Lankford coefficients are compared to those which are identified by a different strategy.
\end{abstract}

Keywords: anisotropy, strategy, identification, behavior law, off-axis testing

\section{Introduction}

Except for certain processes such as molding, a large majority of metal parts is obtained by forming processes during which the material is plastically deformed. They are optimized to reduce the cost, which requires manufacturers to increasingly use numerical simulation and, therefore, need to describe the material behavior.

These simulations are often flawed by a simplified description of the plastic behavior of the material; particularly the anisotropy of rolled sheets (Ghouati and Gelin, 2001; Znaidi, 2004; Haddadi et al., 2006; Kim et al., 2007; Barlat and Lian, 1989). Therefore, it is important to accurately model the plastic behavior of metals in large deformation in order to better predict their behavior of the part during the forming processes (Boubakar and Boisse, 1998; Manget and Perre, 1999; Dogui, 1989).

To describe the plastic behavior of the material, it is necessary to clarify the concept of the load surface related to a plasticity criterion (Barlat and Brem, 1991) that indicates conditions of plastic flow.

The experimental determination of these areas through various mechanical testing and mathematical modeling has been the subject of many inquisitive efforts such as those using the Von Mises criterion because of its implementation in most commercial finite element codes. This criterion is called the energy criterion in which the elastic deformation energy of the material must not exceed a limit value to remain within the elastic range. 
In the case of a metal sheet, the material is considered as having orthotropic plasticity where it reserves three preferred directions as it is used in the Hill criterion (Hill, 1948).

Also, to describe the asymmetric behavior in tension and compression such as the anisotropy of a compact hexagonal structure of a metal sheet, Cazacu et al. $(2008,2009)$ proposed a new orthotropic criterion (Barlat and Lian, 1989; Gronostajsk, 2000; Plunkett et al., 2006).

The objective of this work is to provide a model for numerical simulation of forming processes by plastic deformation of thin metal sheets. Hence, the importance of developing a general framework for elasto-plastic orthotropic models (initial orthotropic and isotropic hardening) based on the choice of an equivalent stress and using the Barlat criterion especially for aluminum alloys (Barlat and Brem, 1991), a hardening law and a plastic potential (Znaidi et al., 2009; Baganna et al., 2010). An identification of the model parameters from an experimental database is developed.

This database consists of many hardening curves from various tests interpreted as homogeneous (Znaidi, 2004) and their Lankford coefficients (Lankford et al., 1950). Those plates are obtained from a hot-rolling process. We use in our work a method called the Simplex algorithm for computer programming to identify the constitutive parameters of the material behavior. This is an important step. A new identification strategy with its validation will be presented, followed by a comparative study using the Hill criterion.

\section{Anisotropic elasto-plastic constitutive laws}

The formulation of the anisotropic elasto-plastic behavior in large deformations is well understood now (Znaidi, 2004; Haddadi et al., 2006; Boubakar and Boisse, 1998; Manget and Perre, 1999): using the formalism of the rotating frame ensures the objectivity of the behavior law regardless of the natural (isotropic, anisotropic) constitutive model functions.

In this work, we focus on the plastic hardening behavior. The materials are considered as incompressible with negligible elastic deformations. The plastic hardening constitutive laws that we have to study fall within the following framework (with the stress tensor)

$$
\bar{f}(\overline{\boldsymbol{\sigma}}, \bar{\alpha}) \leqslant 0 \quad \bar{\alpha}=\mathbf{Q}[\alpha]
$$

with $\mathbf{Q}$ being the transformation tensor of Lagrange state to Eulerien state. $\alpha$ represents the internal hardening variable

$$
\overline{\mathbf{D}}=\lambda \bar{h}(\overline{\boldsymbol{\sigma}}, \bar{\alpha}) \quad \dot{\bar{\alpha}}=\lambda \bar{l}(\overline{\boldsymbol{\sigma}}, \bar{\alpha})
$$

with $\lambda$ as the plastic multiplier that can be determined from the consistency condition $\dot{\bar{f}}=0$ and $\overline{\mathbf{D}}$ is the plastic deformation rate tensor.

\section{Orthotropic plasticity model}

Models are formulated for standard generalized materials with isotropic hardening which are described by an internal hardening variable, a law of evolution and an equivalent deformation.

\subsection{Internal variable hardening}

This work is limited to plastic orthotropic behavior. In fact, the material is initially orthotropic and remains orthotropic. The isotropic hardening is assumed to be captured by a single 
scalar internal hardening variable denoted by $\alpha$. In particular, we will assume that the elastic range evolves homothetically, the yield criterion is then written as follows

$$
f\left(\overline{\boldsymbol{\sigma}}^{D}, \alpha\right)=\sigma_{c}\left(\overline{\boldsymbol{\sigma}}^{D}\right)-\sigma_{s}(\alpha)
$$

where $\sigma^{D}$ is the deviator of the Cauchy stress tensor (incompressible plasticity).

The function $\sigma_{c}\left(\overline{\boldsymbol{\sigma}}^{D}\right)$ satisfies the following condition

$$
\sigma_{c}\left(a \overline{\boldsymbol{\sigma}}^{D}\right)=a \sigma_{c}\left(\overline{\boldsymbol{\sigma}}^{D}\right) \quad \text { for all } \quad a>0
$$

This property implies that the normal $\overline{\mathbf{n}}_{c}=\partial \sigma_{c} / \partial \overline{\boldsymbol{\sigma}}^{D}$ is homogeneous of the zero degree with respect to $\overline{\boldsymbol{\sigma}}^{D}$

$$
\begin{array}{ll}
\overline{\mathbf{n}}_{c}\left(a \overline{\boldsymbol{\sigma}}^{D}\right)=\overline{\mathbf{n}}_{c}\left(\overline{\boldsymbol{\sigma}}^{D}\right) \quad \text { for all } \quad a>0 \\
\overline{\boldsymbol{\sigma}}^{D}: \overline{\mathbf{n}}_{c}=\sigma_{c}
\end{array}
$$

\subsection{Evolution law}

We assume the existence of a plastic potential $g\left(\overline{\boldsymbol{\sigma}}^{D}, \sigma_{s}(\alpha)\right)$ as follows

$$
g\left(\overline{\boldsymbol{\sigma}}^{D}, \sigma_{s}(\alpha)\right)=\sigma_{p}\left(\overline{\boldsymbol{\sigma}}^{D}\right)-\sigma_{s}(\alpha)
$$

The hardening function $\sigma_{s}(\alpha)$ plays the role of the thermodynamic function associated with the internal hardening variable $\alpha$.

The function $\sigma_{p}\left(\overline{\boldsymbol{\sigma}}^{D}\right)$ is assumed to beorthotropic positively homogeneous in the first degree with respect to $\overline{\boldsymbol{\sigma}}^{D}$. The evolution law can be written as follows

$$
\overline{\mathbf{D}}=\lambda \overline{\mathbf{n}}_{p} \quad \overline{\mathbf{n}}_{p}=\frac{\partial \sigma_{p}}{\partial \overline{\boldsymbol{\sigma}}^{D}} \quad \dot{\alpha}=-\lambda \frac{\partial g}{\partial \sigma_{s}}=\lambda
$$

The hypothesis of associated plasticity implies that the plastic potential $g$ is identically $\sigma_{c}$.

The behavior model will be defined by data from two equivalent stresses, $\sigma_{c}$ and $\sigma_{p}$, which are unique and similar. We consider the case of non-associated normality.

\subsection{Equivalent deformation}

The equivalent deformation is obtained according to one of the following definitions:

— Equivalent deformation under the criterion $\varepsilon_{c}$

$$
\dot{\varepsilon}_{c}=\frac{1}{\sigma_{c}}\left(\overline{\boldsymbol{\sigma}}^{D}: \overline{\mathbf{D}}\right)=\frac{1}{\sigma_{c}}\left(\boldsymbol{\sigma}^{D}: \mathbf{D}\right)=\frac{\lambda \sigma_{p}}{\sigma_{c}}
$$

- Equivalent deformation according to the potential $\varepsilon_{p}$

$$
\dot{\varepsilon}_{p}=\frac{1}{\sigma_{p}}\left(\overline{\boldsymbol{\sigma}}^{D}: \overline{\mathbf{D}}\right)=\frac{1}{\sigma_{p}}\left(\boldsymbol{\sigma}^{D}: \mathbf{D}\right)=\lambda
$$

\section{Orthotropic equivalent stresses}

Any orthotropic function $\sigma_{c}\left(\overline{\boldsymbol{\sigma}}^{D}\right)$ with respect to $\boldsymbol{\sigma}^{D}$ is written in a general way according to the following invariant:

— isotropy: $\operatorname{det}\left(\boldsymbol{\sigma}^{D}\right),\left|\boldsymbol{\sigma}^{D}\right|$

- transverse isotropy: $\boldsymbol{\sigma}^{D}: \mathbf{m}_{3},\left(\boldsymbol{\sigma}^{D}\right)^{2}: \mathbf{m}_{3}$

- orthotropy: $\boldsymbol{\sigma}^{D}:\left(\mathbf{m}_{1}-\mathbf{m}_{2}\right), \sqrt{\left(\boldsymbol{\sigma}^{D}\right)^{2}:\left(\mathbf{m}_{1}-\mathbf{m}_{2}\right)}$ 
where $\mathbf{m}_{i}=\vec{m}_{i} \otimes \vec{m}_{i}$ (without summation), $\vec{m}_{i}$ being the orthonormal orthotropy landmark.

The application of this configuration to the stress deviator gives us

$$
\begin{aligned}
& \bar{x}_{1}=\sqrt{\frac{3}{2}}\left(\sigma_{11}^{D}+\sigma_{22}^{D}\right) \quad \bar{x}_{2}=\frac{1}{\sqrt{2}}\left(\sigma_{11}^{D}-\sigma_{22}^{D} \quad \bar{x}_{3}=\sqrt{2} \sigma_{12}^{D}\right. \\
& \bar{x}_{4}=\sqrt{2} \sigma_{23}^{D} \quad \bar{x}_{5}=\sqrt{2} \sigma_{13}^{D}
\end{aligned}
$$

Using the special setup of the space deviators, we can write the stress deviator as follows

$$
\begin{array}{ll}
\left|\boldsymbol{\sigma}^{D}\right|=\sqrt{x_{1}^{2}+x_{2}^{2}} & \operatorname{det}\left(\boldsymbol{\sigma}^{D}\right)=\frac{x_{1}}{\sqrt{6}}\left(x_{2}^{2}-\frac{x_{1}^{2}}{3}\right) \\
x_{1}=\sqrt{\frac{3}{2}}\left(\sigma_{1}^{D}+\sigma_{2}^{D}\right) & x_{2}=\frac{1}{\sqrt{2}}\left(\sigma_{1}^{D}-\sigma_{2}^{D}\right)
\end{array}
$$

We introduce the angle $\theta$ which defines the orientation of $\boldsymbol{\sigma}^{D}$ in the deviatory plane

$$
x_{1}=\left|\boldsymbol{\sigma}^{D}\right| \cos \theta \quad x_{2}=\left|\boldsymbol{\sigma}^{D}\right| \sin \theta
$$

We define the off-axis angle $\psi$ (angle which defines the orientation of the loading directions with respect to the preferred direction of the material) as in the following

$$
\bar{x}_{1}=x_{1} \quad \bar{x}_{2}=x_{2} \cos 2 \psi \quad \bar{x}_{3}=x_{2} \sin 2 \psi
$$

This allows us to write them in terms of the two angles $\theta$ and $\psi$

$$
\bar{x}_{1}=\left|\boldsymbol{\sigma}^{D}\right| \cos \theta \quad \bar{x}_{2}=\left|\boldsymbol{\sigma}^{D}\right| \sin \theta \cos 2 \psi \quad \bar{x}_{3}=\left|\boldsymbol{\sigma}^{D}\right| \sin \theta \sin 2 \psi
$$

Using the special setup of the space deviators, the general form of the equivalent orthotropic plane constraint, is thus

$$
\sigma_{c}\left(\overline{\boldsymbol{\sigma}}^{D}\right)=\sigma_{c}\left(\bar{x}_{1}, \bar{x}_{2},\left|\bar{x}_{3}\right|\right)=\frac{\left|\overline{\boldsymbol{\sigma}}^{D}\right|}{f(\theta, 2 \psi)}
$$

Any type of criterion (4.6) can be written in the form

$$
f(\theta, 2 \psi)=\frac{\left|\overline{\boldsymbol{\sigma}}^{D}\right|}{\sigma_{s}(\alpha)}
$$

where $\theta$ is the angle that defines the test and $\psi$ the off-axis angle (Baganna et al., 2010; Lankford et al., 1950).

Table 1. Values of $\theta$ relative to various tests

\begin{tabular}{|c|c|c|c|c|}
\hline Test & $\begin{array}{c}\text { Expansions } \\
\text { equibiaxes }\end{array}$ & $\begin{array}{c}\text { Simple } \\
\text { traction }\end{array}$ & $\begin{array}{c}\text { Large } \\
\text { traction }\end{array}$ & $\begin{array}{c}\text { Simple } \\
\text { shear }\end{array}$ \\
\hline \hline$\theta$ & 0 & $\pi / 3$ & $\pi / 6$ & $\pi / 2$ \\
\hline
\end{tabular}

\section{Identification procedures}

\subsection{Basic assumption}

In this Section, we focus on the phenomenology of plastic behavior; especially modeling plasticity and hardening based on experimental data represented as families of hardening curves, and Lankford coefficient data. In order to simplify our identification process, the following assumptions are adopted: 
- Hypothesis 1 - Identification through "small perturbations" process.

- Hypothesis 2 - The tests used are considered as homogeneous tests.

- Hypothesis 3 - We neglect the elastic deformation; the behavior is considered as rigid plastic incompressible.

- Hypothesis 4 - The plasticity surface evolves homothetically (isotropic hardening).

- Hypothesis 5 - All tests are performed in the plane of the sheet resulting in a plane stress condition.

\subsection{Limitation of the model}

The identified model is defined by:

- An equivalent stress $\sigma_{c}\left(\mathbf{A}: \boldsymbol{\sigma}^{D}\right), \sigma_{c}$ is an isotropic function. It is assumed that the shape is defined by coefficients of the form $m_{i}$.

A - 4th order orthotropic tensor defined by anisotropy coefficients $a_{i}$.

- A potential equivalent stress $\sigma_{p}\left(\mathbf{A}_{p}: \boldsymbol{\sigma}^{D}\right), \sigma_{p}$ is defined by coefficients of the form $m_{i}^{\prime}$ and anisotropic coefficients $a_{i}^{\prime}$.

- Hardening curve $\sigma_{s}(\alpha)$

The tests used for the identification of this model are "radial" and "monotonous" tests

$$
\sigma=\sigma \mathbf{a}
$$

with $\sigma>0$ and increasing, and $\mathbf{a}$ is constant.

And the deformation tensor is

$$
\varepsilon=\varepsilon \mathbf{b}
$$

Knowing that $\sigma(\varepsilon)$ is determined from experimental tests as $r(\psi)$.

According to the yield criterion (3.1)

$$
\sigma_{c}(\mathbf{q})-\sigma_{s}(\alpha) \leqslant 0
$$

where $\mathbf{q}=\mathbf{A}\left(a_{i}\right): \boldsymbol{\sigma}$, thus $\mathbf{q}=\sigma \mathbf{A}\left(a_{i}\right): \mathbf{a}$.

So the equivalent stress (positively homogeneous of one degree)

$$
\sigma_{c}(\sigma \mathbf{A}: \mathbf{a})=\sigma \sigma_{c}(\mathbf{A}: \mathbf{a})=\sigma a_{e}
$$

The equivalent deformation is determined from the duality relation

$$
\dot{\varepsilon}_{c}=\frac{\sigma \dot{\varepsilon}}{\sigma_{c}}
$$

Similar to the equivalent deformation relative to the potential, we write

$$
\varepsilon_{p}=\varepsilon b_{e}
$$

When the potential identifies the criterion, we have $b_{e}=1 / a_{e}$.

Under these conditions, our hardening curve may be written as in the following

$$
\sigma a_{e}=\sigma_{s}\left(\varepsilon b_{e}\right) \Rightarrow \sigma=\frac{\sigma_{s}\left(\varepsilon b_{e}\right)}{a_{e}}
$$




\section{First comment}

If our hardening curve is shown by an analytical law such as the following law

$$
\begin{aligned}
& \sigma_{s}=k\left(\alpha_{0}+\alpha\right)^{n} \Rightarrow \sigma=\frac{k\left(\alpha_{0}+\varepsilon b_{e}\right)^{n}}{a_{e}}=k_{\text {test }}\left(\alpha_{\text {test }}+\varepsilon\right)^{n} \\
& k_{\text {test }}=\frac{k b_{e}^{n}}{a_{e}} \quad \alpha_{\text {test }}=\frac{\alpha_{0}}{b_{e}}
\end{aligned}
$$

when $n$ is the same for all tests.

A second analytical law

$$
\begin{aligned}
& \sigma_{s}=\sigma_{0}+k \alpha^{n} \Rightarrow \sigma=\frac{\sigma_{0}+k\left(\varepsilon b_{e}\right)^{n}}{a_{e}} \\
& \sigma_{s}=\sigma_{0 \text { test }}+k_{\text {test }} \varepsilon^{n} \quad \text { with } \quad \sigma_{0 \text { test }}=\frac{\sigma_{0}}{a_{e}} \quad k_{\text {test }}=\frac{k b_{e}}{a_{e}}
\end{aligned}
$$

In conclusion, we can say that regardless of the analytical law representing the hardening curve and whatever the test, the value of $n$ does not change.

\section{$\underline{\text { Second comment }}$}

We can also begin our identification procedures using the Lankford coefficient as determined from the tensile test by

$$
r=\frac{\dot{\varepsilon}_{2}}{\dot{\varepsilon}_{3}}=-\frac{1}{1+\dot{\varepsilon}_{1} / \dot{\varepsilon}_{2}}
$$

We can notice that the Lankford coefficient is independent of $\varepsilon$. This coefficient is equal to one in the case of isotropy, and remains constant in the case of transverse isotropy. However, in the case of orthotropy, it varies depending on the off-axis angle $\psi$. This coefficient completely characterizes the anisotropy of the sheet when loaded in its plane

$$
\dot{\varepsilon}=\lambda \mathbf{n}_{p}(\sigma \mathbf{a}) \quad a_{T \psi}=\frac{\dot{\varepsilon}_{1}}{\dot{\varepsilon}_{2}}=\frac{\left[n_{p}(a)\right]_{11}}{\left[n_{p}(a)\right]_{22}} \quad r(\psi)=-\frac{1}{1+a_{T \psi}}
$$

So identifying this coefficient, means determining $a_{T \psi}$ relative to a well chosen model.

\section{Results and discussion}

This identification strategy requires: (a) an experimental database, (b) criterion for anisotropic plasticity and (c) validation strategy.

In the particular case of aluminum sheets, where anisotropy is present, the identification of this constitutive law requires the identification of:

- The hardening coefficients $k$ and $n$,

- The anisotropy coefficients $f, g, h$, and $n$ notes that $a_{i}$ has the form factor of $m$,

- The Lankford coefficient $r(\psi)$.

The test specimens are cut in different directions relative to the rolling direction of the sheet, according to the geometry defined in Fig. 1. 


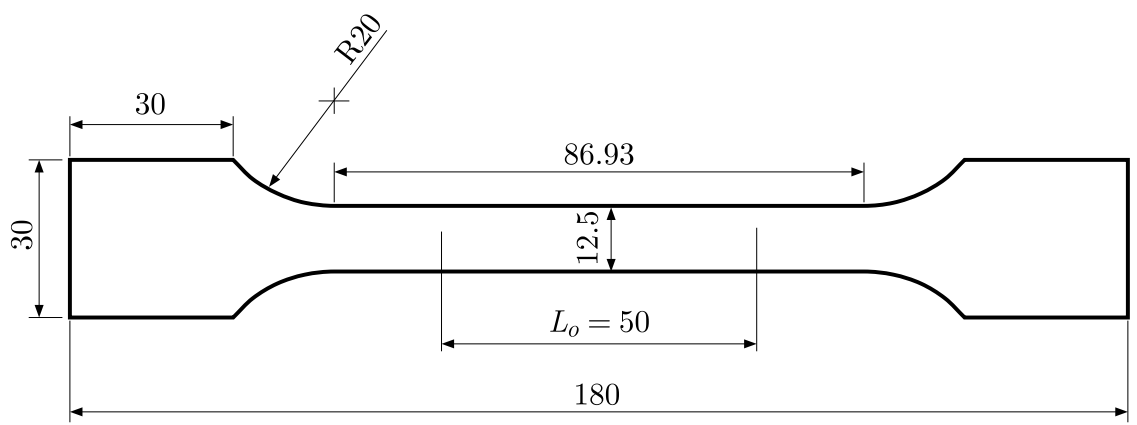

Fig. 1. Test piece in tension. Definition of the systems axis and current dimensions of the active area $\left(b_{0}=12.5 \mathrm{~mm}\right)$

\subsection{Identification of $k$ and $n$}

Using the Hollomon law

$$
\sigma_{s}=k \varepsilon^{n}
$$

And using the Barlat criterion (Barlat and Brem, 1991)

$$
\sigma_{c}^{m}=\left|q_{1}-q_{2}\right|^{m}+\left|q_{2}-q_{3}\right|^{m}+\left|q_{1}-q_{3}\right|^{m}
$$

where $q_{1}, q_{2}$ and $q_{3}$ are the eigenvalues of the tensor $\mathbf{q}$ defined by Eq. (5.3).

Using the plastic Barlat model and respecting the assumptions, the identification of the thin aluminum sheet is equivalent to choosing the coefficients of the model while minimizing the squared difference between the theoretical and experimental results.

In Table 2, we present the values of $k$ and $n$ for different tractions tests.

Table 2. Identification of the constants of the hardening law

\begin{tabular}{|l|c|c|c|}
\hline$\psi$ & $0^{\circ}$ & $45^{\circ}$ & $90^{\circ}$ \\
\hline$K\left[\mathrm{daN} / \mathrm{mm}^{2}\right]$ & 15.1117 & 17.2835 & 13.8759 \\
\hline$n$ & 0.253 & 0.2316 & 0.2485 \\
\hline
\end{tabular}

Knowing that the coefficient $n$ is the same for all tests as demonstrated at the beginning of this work. By convention we choose $n$ for traction in the direction $\psi=0^{\circ}$ as a reference. For $n=0.253$, we present different values of $k$ (Table 3 ).

Table 3. Identification of the constant hardening law for the fixed $n$

\begin{tabular}{|l|c|c|c|}
\hline$\psi$ & $0^{\circ}$ & $45^{\circ}$ & $90^{\circ}$ \\
\hline$K\left[\mathrm{daN} / \mathrm{mm}^{2}\right]$ & 15.1132 & 18.0828 & 14.002 \\
\hline
\end{tabular}

In Figs. 2a and 2b, the experimental hardening curves (exp) and the curves identified from the model (ident1) using an average value of $n$ and the curves identified by our model (ident2) are represented. For tensile tests in $\psi=45^{\circ}$, the two models (ident1) and (ident2) give a clear fit between the theoretical and experimental results. However, for tests in $\psi=0^{\circ}$, identifying these results provides better validation for a good agreement between the experimental and theoretical results for the model (ident2) presented in this work.

\subsection{Identification of anisotropic coefficients $a_{i}$ and shape coefficient $m$}

Our second identification determines the coefficients of anisotropy $(f, g, h, n)$ and the shape coefficient $m$ (Table 4), considering the Barlat criterion (6.2). 

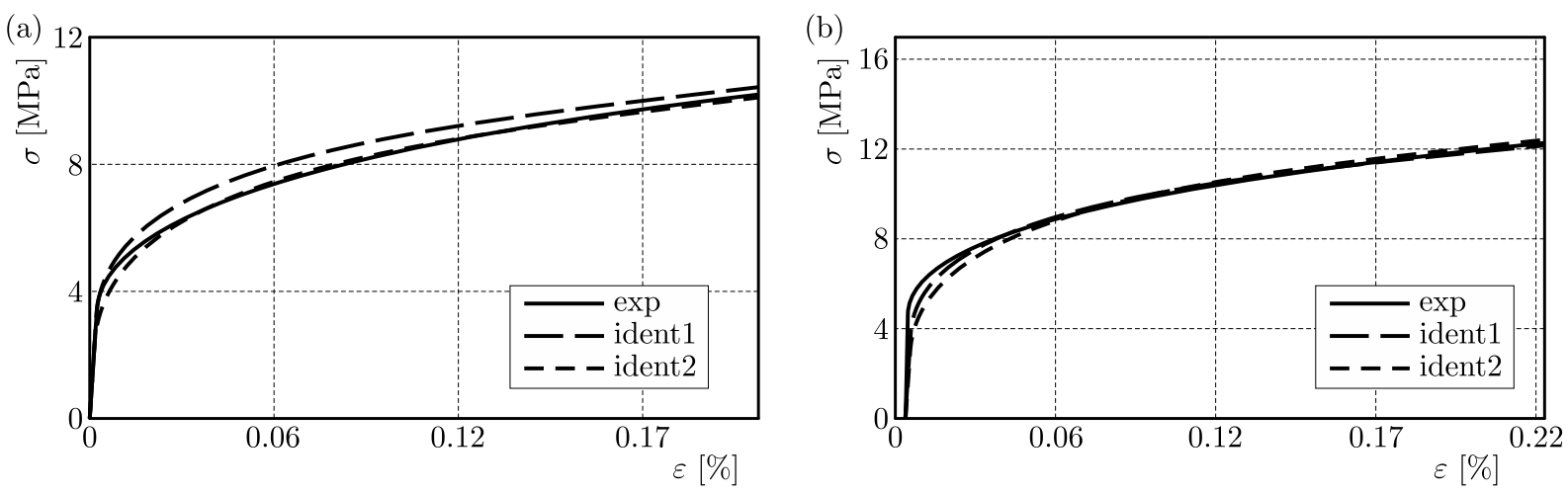

Fig. 2. Identification of the hardening curve: (a) $\psi=0^{\circ}$, (b) $\psi=45^{\circ}$

Table 4. Identification of anisotropic coefficients and the shape coefficient $m$

\begin{tabular}{|c|c|c|c|c|}
\hline$F$ & $g$ & $h$ & $n$ & $m$ \\
\hline \hline 0.2854 & 0.2064 & 0.3335 & 0.8921 & 6.9584 \\
\hline
\end{tabular}

\subsection{Evolution of the anisotropic and the Lankford coefficient}

Using the identified anisotropic coefficients, we represent in Fig. 3a the developments of the Lankford coefficient and the evolution of the anisotropy based on off-axis angles (Fig. 3b).

(a)

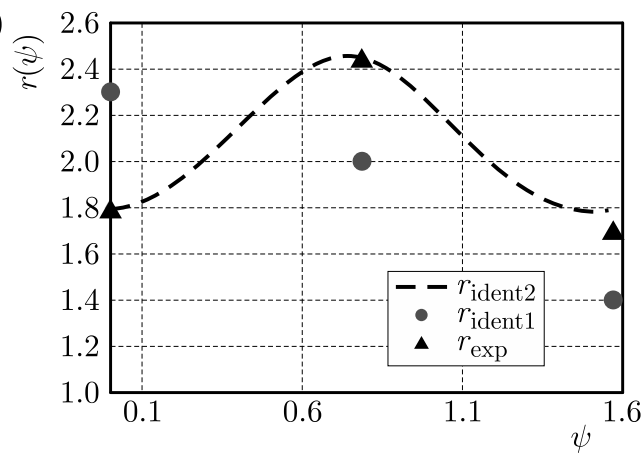

(b)

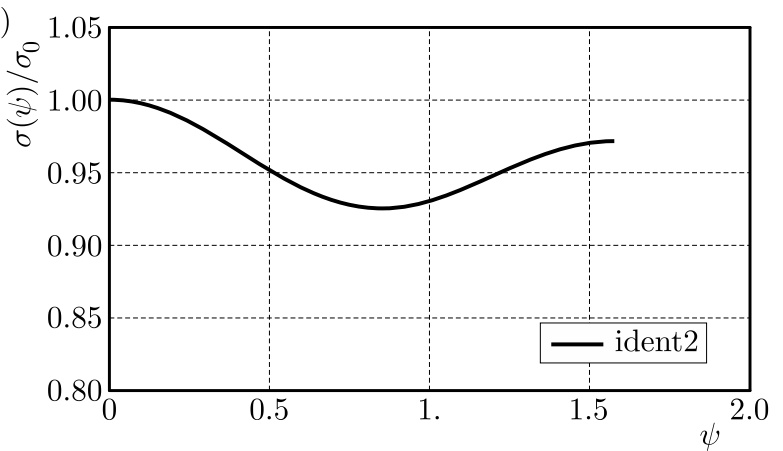

Fig. 3. (a) Evolution of the Lankford coefficient and (b) evolution of the anisotropy based $\psi$

We find a good agreement between the experimental results and those from the model using the Barlat criterion. We notice an important anisotropy at $\psi=45^{\circ}$ (see Fig. 3b). However, the identification using the Hill criterion is not validated by the Lankford coefficient.

\subsection{Evolution of the load surface}

The model (ident2) allows us to study the load surface on each test. We note that this material is resistant to simple shear much more than to simple traction. In contrast, the plastic flow in wide traction (i.e. the specimen length is comparable to its width) is quickly reached.

\section{Validation}

We use tensile tests at $\psi=90^{\circ}$ in order to validate our model. The results show a good agreement between the theoretical results on the model (using anisotropic coefficients, shape coefficient $m$ ) and experimental data (Fig. 5). 


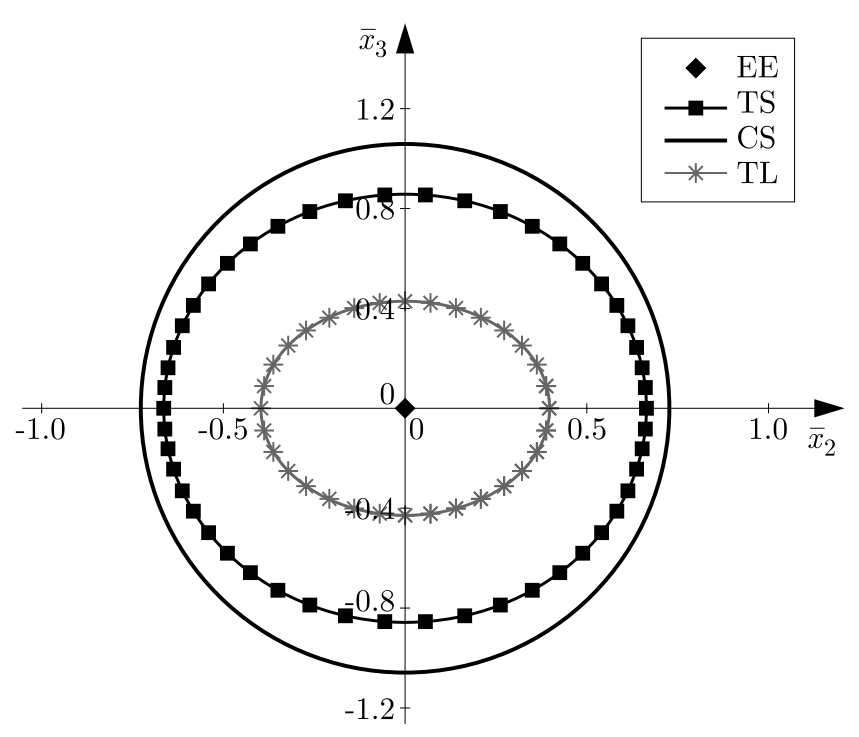

Fig. 4. Evolution of the load surface in the deviatory plane $\left(\bar{x}_{2}, \bar{x}_{3}\right)$

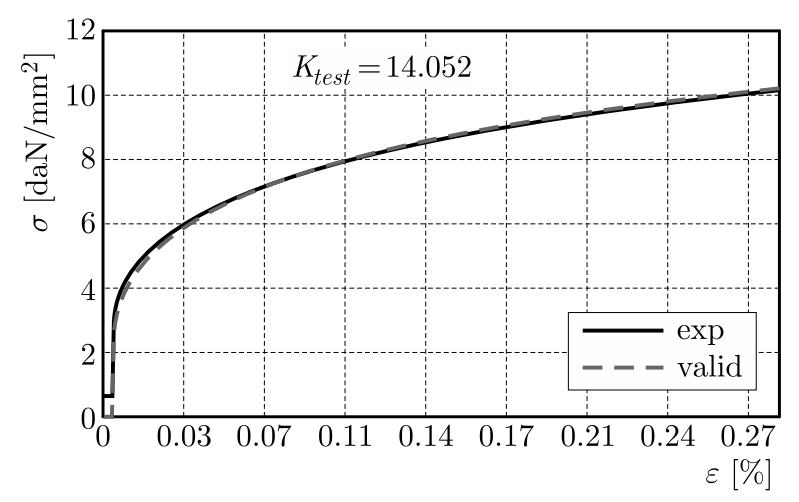

Fig. 5. Validation of the hardening curve at $\psi=90^{\circ}$

\section{Conclusion}

In this work, we show that the identification strategy results can be extracted. This identification has focused both on plastic material parameters of the constitutive law and the Lankford coefficients. Thus, the plastic behavior model: the Hollomon law and the Barlat criterion with 5 parameters are identified.

Validation by comparing the models with the experiment data base has been performed.

The model using the Barlat criterion is in good agreement with the experimental results relating to the Lankford coefficient. It is better than the Hill model.

Following this strategy, we observe very pronounced anisotropy of Aluminum A5 and the load surface for different tests at the end of this identification.

\section{Reference}

1. Baganna M., Znaidi A., Kharroubi H., Nasri R., 2010, Identification of Anisotropic Plastic Behavior Laws for Aluminum 2024 after Heat Treatment Materials from Off-Axis Testing, Toulouse

2. Barlat F., Brem D.L.J., 19991, A six components yield function for anisotropic materials, International Joural of Plasticity, 7, 693-712 
3. Barlat F., Lian J., 1989, Plastic behavior and stretchability of sheet metals. Part I: A yield function for orthotropic sheet under plane stress conditions, International Joural of Plasticity, 5, $51-56$

4. Boubakar L., Boisse P., 1998, Anisotropic elastoplastic behavior for numerical analysis of thin shells in great transformations, Européene Review Mechanics, 7, 6

5. Cazacu O., Ionescu I.R., Yoon J.W., 2009, Orthotropic strain rate potential for the description of anisotropy in tension and compression of metals, International Joural of Plasticity

6. Cazacu O., Plunkett B., Barlat F., 2008, Orthotropic yield criterion description of anisotropy in tension and compression of sheet metals, International Joural of Plasticity, 24, 847-866

7. Dogui A., 1989, Anisotropic plasticity in large deformation, Doctoral thesis of sciences, University Claude Bernard-Lyon

8. Ghounti O., Gelin J.C., 2001, A finite element based identification method for complex metallic material behavior, Computational Materials Science, 2157-2168

9. Gronostajsk Z.. 2000, The constitutive equations for FEM analysis, Journal of Material Processing Technology, 106, 40-44

10. Haddadi H., Bouvier S., Banu M., Maier C., Teodosiu C., 2006, Towards an accurate description of the anisotropic behaviour of sheet metals under large plastic deformations: modelling, numerical analysis and identification, International Joural of Plasticity, 22, 2226-2271

11. Hill R., 1948, A theory of the yielding and plastic flow of anisotropic metals, Proceedings of Royal Society of London, A93, 281-297

12. Kim D., Barlat F., Bouvier S., Rabahallah M., Balan T., Chung K., 2007, Non-quadratic anisotropic potential based on linear transformation of plastic strain rate, International Joural of Plasticity, 23, 1380-1399

13. LAnkford W.I., Snyder S.C., Bausher J.A., 1950, New criteria for predicting the press performance of deep- drawing sheets, Transactions of American Society for Metals, 42, 1196-1232

14. Manget B., Perre P., 1999, A large displacement formulation for anisotropic constitutive laws, European journal of Mecanique, 18, 5

15. Plunkett B., Cazacu O., Barlat F., 2006, Orthotropic yield criterion for hexagonal closed packed metals, International Joural of Plasticity, 22, 1171-1194

16. ZnAidi A., 2004, Plasticity orthotrope in large deformation». PhD thesis at the Faculty of Sciences of Tunis

17. Znaidi A., Soula M., Guellouz S., NAsri R., 2009, Constitutive Equations Identification Strategies Elastoplastic Anisotropics Heet Material Using Associated and Unassociated Laws of Plasticity, Marseille 